\title{
Von den Blattübersichten schweizerischer Landeskartenwerke zur Titelaufnahme im Bibliotheksverbundkatalog
}

\author{
Thomas Klöti
}

In den Bibliotheksverbundkatalogen erfolgt eine Recherche bisher immer verbal. Dies mag für Bücher die einzig richtige Lösung sein, für Karten hingegen ist eine derartige Suchstrategie oft kaum aussagekräftig. Zu den lang gehegten Wünschen im Kartenbibliothekswesen gehört daher eine graphische Suchoberfläche, die eine geographische Suche bzw. eine Suche nach Koordinaten ermöglicht.

\section{Von der Blattübersicht in den Bibliotheksverbundkatalog}

Eine Zwischenlösung im Hinblick auf eine textfreie, graphische Suche stellt der Einsatz von Blattübersichten für Kartenwerke dar. Die Kartensammlung der ETHBibliothek behilft sich zur Zeit mit Blattübersichten, denen eine im HTML-Format zusammengestellte Liste der vorhandenen Ausgaben hinterlegt ist $<$ http://www.ethbib.ethz.ch/ks/indexblaetter.html>. Der Link von der Blattübersicht zur HTML-Seite erfolgt dabei aufgrund von verweis-sensitiven Graphiken, die auch als image maps bzw. als clickable maps bezeichnet werden. Für die Kartensammlung des Bundesamts für Landestopographie in Wabern bei Bern (L+T) $<$ http://www.swisstopo.ch/de/maps/ak/INDEX.htm> haben wir nun verweis-sensitive Blattübersichten mit einem direkten Link in den seit März 2000 öffentlich zugänglichen Bibliotheksverbundkatalog der schweizerischen Bundesverwaltung <http://auriga.admin.ch:8081/> geschaffen.

Der Beginn der Katalogisierung der Kartensammlung bzw. des Projekts Katalog Kartenarchiv der L+T erfolgte am 1. April 1999. Die Kartensammlung umfasst insgesamt zirka 23.000 handgezeichnete Blätter und Unikate sowie zirka 29.000 gedruckte Karten (ohne Mehrfachexemplare). Bis Ende 2000 wurden unter anderem sämtliche Ausgaben der schweizerischen Landeskartenwerke erfasst, die auch unter den Bezeichnungen „Dufourkarte“, „Siegfriedkarte“ und „Landeskarte“ bekannt sind. Insgesamt wurden bis zu diesem Zeitpunkt 10.846 Katalogeinträge vorgenommen und mit den Mehrfachexemplaren insgesamt 67.611 Kartenblätter nachgewiesen. Im Produktekatalog des Bundesamts für Landestopographie wird die Kartensammlung unter den Dienstleistungen aufgeführt. Im Hinblick auf die Neugestaltung der Webseiten der Kartensammlung der L+T, die im März 2001 im Internet zugänglich gemacht wurden <http://www.swisstopo.ch/de/maps/ak/INDEX.htm>, war es uns ein Anliegen, den internen und externen Benutzerinnen und Benutzern dieser Dienstleistung einen leicht verständlichen und zielgerichteten Zugang zu diesen Kar- 
tenbeständen zu schaffen. Damit wird die selbständige Recherche in der Datenflut eines Bibliothekskatalogs, der von mehr als 30 Verbundbibliotheken gespiesen wird, wesentlich erleichtert. Die verweis-sensitiven Blattübersichten dienen aber auch dem Betreuer der Kartensammlung, dem zusätzlich zum Katalog ein weiteres Arbeitsinstrument in die Hand gegeben wird, um Kundinnen und Kunden schnell und zielgerichtet beraten zu können.
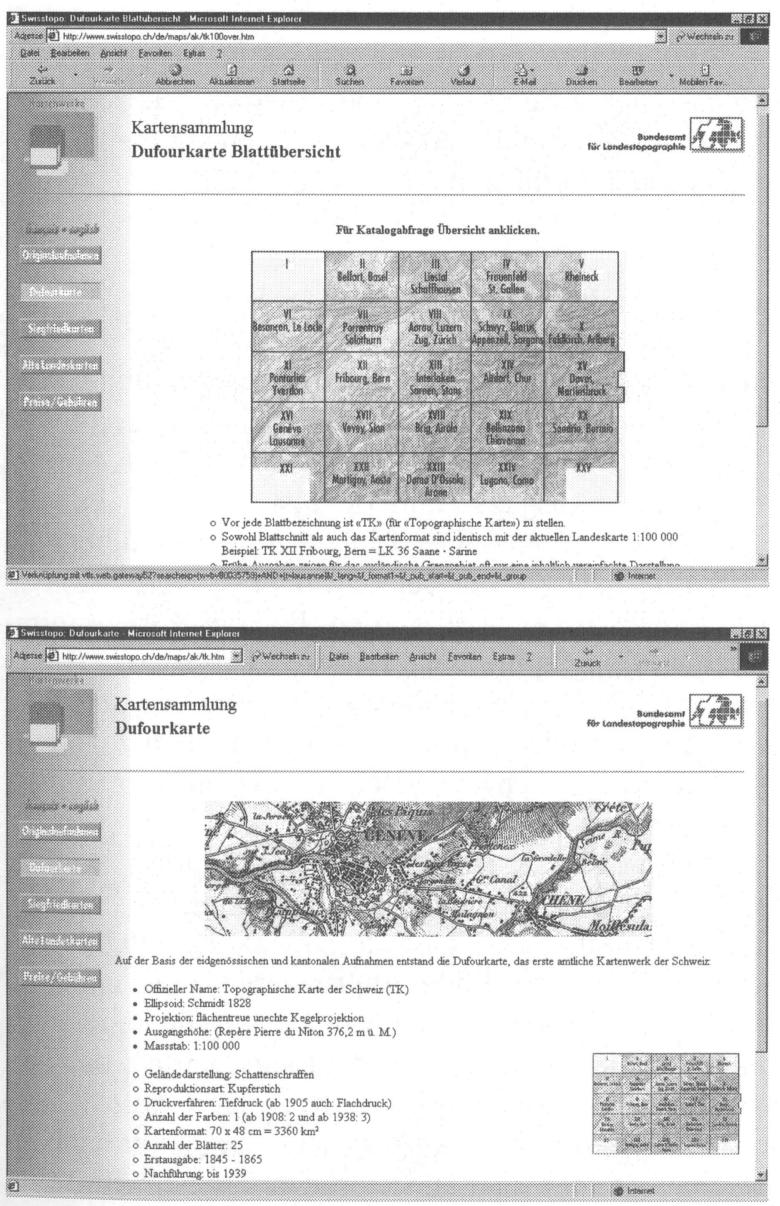

Abb. 1: Die Kartensammlung des Bundesamts für Landestopographie $(L+T)$ bewahrt mehr als 50.000 Karten auf, wozu auch sämtliche Ausgaben der amtl. Kartenwerke gehören (Dufourkarte, Siegfriedkarte, Landeskarte)

Abb.2: Textfreie, graphische Katalogabfrage in den Beständen der Kartensammlung des Bundesamts für Landestopographie mit einer verweis-sensitiven Blattübersicht der Dufourkarte 1:100.000

Aufgrund der engen Zusammenarbeit mit den zuständigen Fachpersonen innerhalb der $\mathrm{L}+\mathrm{T}$ konnte dieser graphische Zugriff auf den Bibliothekskatalog innert der von uns gesetzten kurzen Frist entwickelt und nach einer kurzen Testphase für die $\mathrm{Be}-$ nutzung im Internet freigegeben werden. 

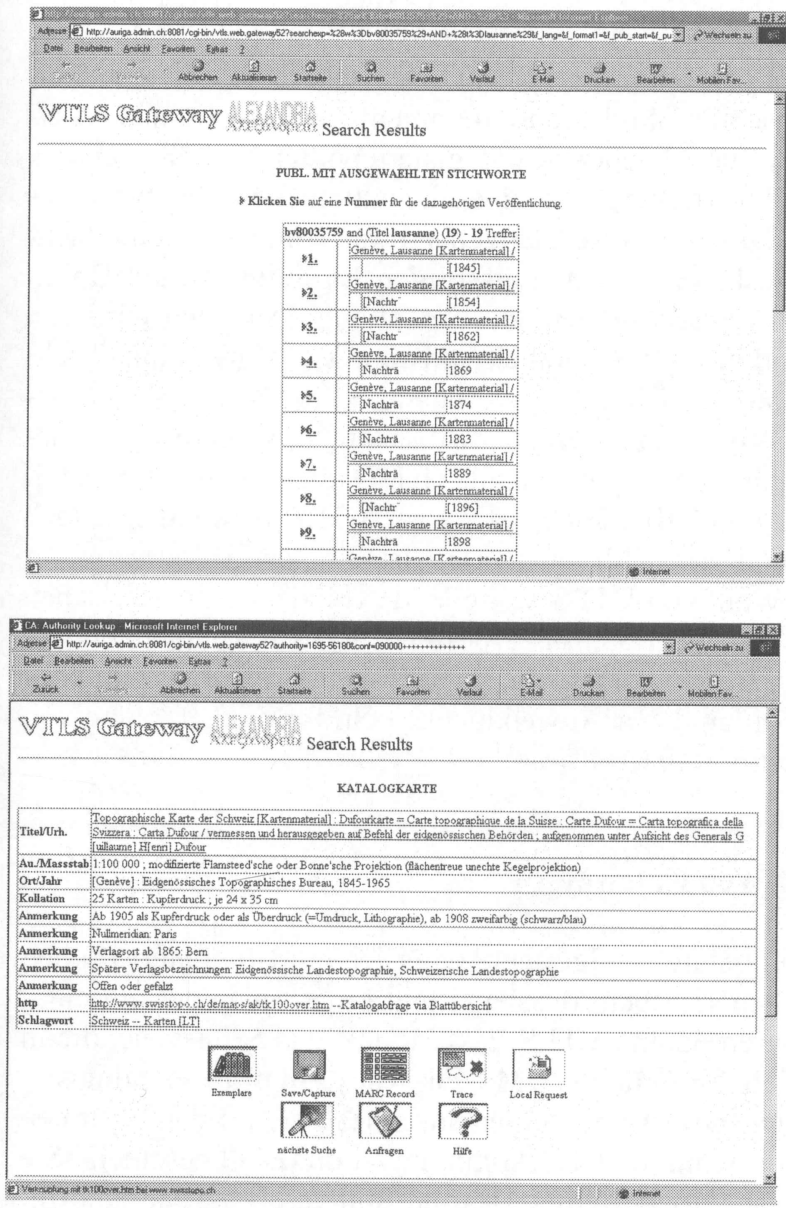

Abb. 3: Suchresultat mit der Vorauswahl für die im Bibliotheksverbundkatalog Alexandria vorhandenen und über das VTLS Web Gateway abfragbaren Ausgaben von Blatt 16 der Dufourkarte 1:100.000 der Kartensammlung

Abb. 4: Die graphische „Katalogabfrage via Blattübersicht" kann für die Blätter der Dufourkarte (,,Topographische Karte der Schweiz 1:100.000") direkt vom Bibliotheksverbundkatalog aus aktiviert werden

\section{Der Bibliotheksverbund Alexandria}

Bevor das Projekt „Katalog Kartenarchiv“ anfang April 1999 gestartet werden konnte, musste in einem Vorprojekt die Software für die Erschliessung der Sammlung evaluiert werden. Wir gingen von der Grundannahme aus, dass eine verbundtaugliche Bibliothekssoftware eingesetzt werden soll. Bewusst wurde damit auf den Einsatz einer „Insellösung“ verzichtet. Die Integrierung in einen Bibliotheksverbund wurde insbesondere im Hinblick auf Wartung und Migrierung sowie hinsichtlich der Nutzung von Fremddaten und der Weitergabe eigener Datensätze angestrebt. In die engere Wahl fielen z. B. die damaligen Verbundsysteme ETHICS-plus, Deutsch- 
schweizer Bibliotheksverbund Basel-Bern (DSV) und Alexandria. ETHICS-plus und DSV wechselten in der Zwischenzeit ihr Bibliothekssystem und wuchsen, mit weiteren Hochschulbibliotheken, zum Informationsverbund Deutschschweiz (IDS) sämtlicher Deutschschweizer Hochschulbibliotheken zusammen. Da die erwähnten Verbundsysteme heute im Prinzip die gleichen Katalogisierungsstandards anwenden, gab es keine grundlegenden Unterschiede, die eine eindeutige Bevorzugung eines Bibliothekssystems gerechtfertigt hätte. Ausschlaggebend war schliesslich eine fachtechnische Weisung des Bundeskanzlers zum Einsatz von Bibliotheks- und Dokumentationssystemen in der schweizerischen Bundesverwaltung. Demnach wird von neuen Anwendern die Verwendung der Bibliothekssoftware VTLS (Virginia Technical Library Systems) innerhalb des Bibliotheksverbundes Alexandria als verbindlich erklärt. Von dieser Regel gibt es natürlich auch Ausnahmen, wie die Bibliotheken der Eidgenössischen Technischen Hochschschule (ETH), die sich am IDS beteiligen, oder die Schweizerische Landesbibliothek, die zwar VTLS verwendet, jedoch nicht in den Bibliotheksverbund Alexandria integriert ist. Für das Bundesamt für Landestopographie bringt die Wahl von VTLS Vorteile, da von der Schweizerischen Landesbibliothek bereits existierende Datensätze bezogen werden können. Zudem gibt es auch Synergien mit den Beständen innerhalb der Bundesverwaltung. Im Hinblick auf eine Koordination wurden daher Anwendungsregeln für die Erfassung von Kartenmaterialien ausgearbeitet.

\section{VTLS Easycat, Easypac und Web Gateway}

Die Hauptarbeit für die Erschliessung erfolgt heute natürlich am Computer. $\mathrm{Zu}$ den Arbeitsinstrumenten gehören daher, neben Messband und Lupe sowie der einschlägigen Fachliteratur, die Softwareprodukte VTLS Easycat und VTLS Easypac, die in der Verwendung aufeinander abgestimmt sind. Mit Easycat erfolgt die Katalogisierung auf dem lokalen Computer. Die Daten werden anschliessend auf den zentralen Server beim Bundesamt für Informatik und Telekommunikation (BIT) geschickt. Sie können dann mittels Easypac abgefragt werden. Da die Software Easypac eine Installation erfordert, wird diese eher innerhalb der Bibliotheken eingesetzt, während für den Zugriff via Internetbrowser ein Web Gateway zur Verfügung steht. Die Abfrage kann einerseits im gewohnten Bibliotheksformat stattfinden, anderseits auch im MARC-Format, das heisst so, wie die Datensätze durch den Bibliothekar in den dazu vorgesehenen Feldern erfasst wurden.

Die Karten werden nach den gleichen Regeln wie in der Schweizerischen Landesbibliothek katalogisiert. Kartenmaterialien werden nach den Anglo-American Cataloguing Rules, $2^{\text {nd }}$ ed. (AACR2) erschlossen, wobei für den bibliographischen Datensatz das Format MARC21 Swiss Version in Anwendung kommt. Zusätzlich gibt es Anwendungsregeln für die Schweizerische Landesbibliothek sowie für den Verbund Alexandria, die sich allerdings nur geringfügig voneinander unterscheiden. Im 
Hinblick auf die Frage der Blattübersichten ist es wichtig zu wissen, dass für Kartenmaterial grundsätzlich die Einzelblattkatalogisierung gilt, das heisst, dass jedes einzelne Kartenblatt, sofern es einen eigenen Titel hat, eine bibliographische Aufnahme erhält. Der Link von den Blattübersichten aus dem Internet führt damit direkt auf die jeweiligen bibliographischen Aufnahmen, wobei anschliessend auch sämtliche Funktionalitäten des Bibliothekskatalogs zur Verfügung stehen. Für die Schaffung eines Links vom Bibliothekskatalog auf die Blattübersichten der Kartenwerke wurde zudem das in MARC21 zur Verfügung stehende Feld 856 für elektronische Lokalisierung und Zugriff genutzt. Falls später auch einmal Bilddaten ins Netz gestellt werden sollten, könnte die Verwendung dieses Feldes auch auf der Stufe der Einzelkarten von Nutzen sein.

\section{Umrechungstabelle von Landeskoordinaten in geographische Koordinaten}

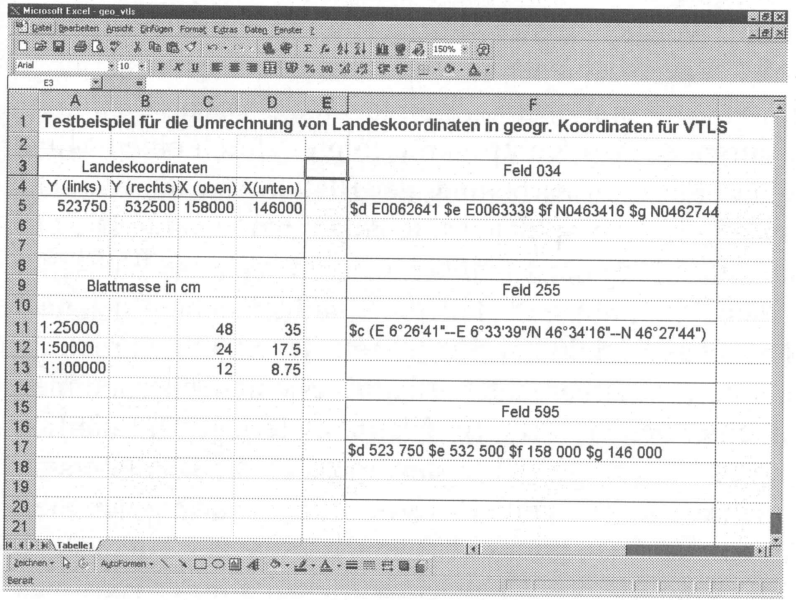

Abb. 5: Umrechnungstabelle für die Umwandlung von Landeskoordinaten in geographische Koordinaten, die anschließend, mit „copy" und "paste", in das $K a$ talogisierungstool VTLS Easycat übertragen werden

Eine wesentliche Beschleunigung der Arbeiten erfolgt durch den Einsatz eines Hilfstools zur Berechnung der geographischen Koordinaten. Die Umrechnung der auf den Blättern der schweizerischen Landeskartenwerke aufgedruckten Landeskoordinaten in geographische Koordinaten geschieht mit einer vom Bereich Geodäsie des Bundesamts für Landestopographie entwickelten Excel-Tabelle. Das Resultat wird in der für die bibliographische Aufnahme erforderlichen Syntax dargestellt, so dass dieses - ohne weitere Nachbearbeitung - mit „copy“ und „paste“ in die dazu vorgesehenen drei Felder von VTLS Easycat übertragen werden kann. Mit einer von uns vorgenommenen Erweiterung können mit dem gleichen Hilfsmittel auch die Masse der Karte für den jeweiligen Maßstab errechnet werden. Der Vorteil liegt nicht nur in der schnelleren Berechnung der Kartenmasse, sondern auch im 
der schnelleren Berechnung der Kartenmasse, sondern auch im Erkennen von Fehlern bei der Eingabe der Landeskoordinaten, die bei nicht plausiblen Ergebnissen vorliegen müssen.

\section{Verweis-sensitive Blattübersichten}

Die Herstellung der Blattübersichten erfolgte, wie einleitend angetönt wurde, in einem mehrteiligen Verfahren, wobei die Bereiche Kartographie, Topographie sowie der Webpublisher des Bundesamts für Landestopographie mitwirkten. Ziel war es, sowohl ein graphisch ansprechendes wie auch ein qualitativ einwandfreies Erzeugnis herzustellen. Vorerst musste geklärt werden, welche bereits bestehende Blattübersicht als Grundlage herangezogen werden soll. Von den schweizerischen Landeskartenwerken sind über die Jahrzehnte immer wieder neue Blattübersichten erschienen, die sich z. B. in ihrer Vollständigkeit aber auch in der Schreibweise für die Blattbezeichnungen unterscheiden. So gibt es z. B. Kartenblätter, die ursprünglich einen deutschsprachigen Titel trugen, welcher später durch eine französischsprachige Blattbezeichnung ersetzt wurde. Wir entschieden uns, jeweils die neueste Blattübersicht für das jeweilige Kartenwerk mit einer stark vereinfachten Basiskarte zu kombinieren. Die redaktionellen Vorgaben und Kontrollen erfolgten durch einen Mitarbeiter des Bereichs Kartographie. Für die Bearbeitung der Blattübersichten von Dufour- und Siegfriedkarte konnte ein Kartographielehrling eingesetzt werden. Die Optimierung für das Internet, die Aufteilung der Blattübersicht auf mehrere Webseiten sowie das Weblayout oblag dem Webpublisher. Für die Blattübersichten der nach wie vor erscheinenden Landeskarten 1:25.000, 1:50.000 und 1:100.000 wurden die bereits bestehenden, für das Internet aufbereiteten Blattübersichten übernommen, vom Webpublisher jedoch mit einer geeigneteren Basiskarte hinterlegt. Um die Lesbarkeit der Blattübersichten im Internet zu gewährleisten, mussten die Blattübersichten großer Kartenwerke auf mehrere Seiten verteilt werden. Dies war bei der Siegfriedkarte 1:25.000 (462 Blätter) und 1:50.000 (142 Blätter) sowie der Landeskarte 1:25.000 (249 Blätter) der Fall. Vom kleinen Übersichtskärtchen auf der Einstiegsseite des jeweiligen Kartenwerks gelangt man zwar bei allen Kartenwerken vorerst auf die gesamte Blattübersicht, bei der Siegfriedkarte 1:25.000 wird man jedoch auf 7, bei der Siegfriedkarte 1:50.000 auf 3 und bei der Landeskarte 1:25.000 auf 4 detailliertere Blattübersichten geführt. Das Navigieren ist jedoch auch hier, mittels des eingefügten Übersichtskärtchens, stets über das gesamte Kartenwerk möglich.

Kernstück der Bearbeitung durch den Webpublisher lag jedoch bei der Aufbereitung der Blattübersichten zu verweis-sensitiven Graphiken (verweis-sensitive Graphiken sind Graphiken, in denen mit der Maus auf ein Detail geklickt werden kann; daraufhin wird ein Verweis bzw. Link ausgeführt). Für sämtliche Rechtecke der jeweiligen Einzelblätter in den Blattübersichten wurden die Eckkoordinaten in HTML-Code 
eingefügt. Dem bibliothekarischen Mitarbeiter oblag es anschließend, die Verweise für sämtliche Einzelblätter der Kartenwerke in diesen sogenannten Quellcode einzufügen. Die für den Verweis verwendete Zeichenkette entspricht derjenigen, die bei einer Expertensuche im Web Gateway generiert wird. (Da die Zeichenkette auf die jetzige Version des Web Gateways zugeschnitten ist, ist zur Zeit nicht absehbar, ob und wann bei künftigen neuen Versionen Anpassungen notwendig werden.) Für das Blatt 16 der Dufourkarte, mit dem Blatttitel „Genève Lausanne“, sieht diese Zeichenkette $\mathrm{z}$. B. folgendermassen aus:

http://auriga.admin.ch:8081/cgi-bin/vtls.web.gateway52?searchexp=\%28w\%3D bv80035759 $\% 29+$ AND $+\% 28 t \% 3$ Dlausane $\% 29 \& f$ _lang $=\& f$ format $1=\& f$ f pub_start $=\& f \_$pub_end $=\& f$ group $=0000 \&$ lang $=$ german

$\mathrm{Zu}$ den wechselnden Teilen der Suchfrage gehören die hier fett gekennzeichneten Elemente, bestehend aus der nur im MARC-Format ersichtlichen IdentifikationsNummer für das Kartenwerk, aus einem Titelwort sowie aus den Bool'schen Operatoren AND, OR oder NOT. Anstelle des im Titel an zweiter Stelle stehenden Wortes „Lausanne“ hätte bei diesem Beispiel auch der String „Gen\%E8ve“ für das an erster Stelle stehende Titelwort „Genève“ eingesetzt werden können („è “ wird dabei zu „\%E8“). Bei der Wahl der Titelworte musste zudem auf allerlei Besonderheiten Rücksicht genommen, damit einerseits alle Blätter und andererseits nur Blätter des jeweiligen Kartenblattes im Suchresultat erscheinen. Mit dem folgenden Beispiel soll dies verdeutlicht werden: Um alle in der Kartensammlung vorhandenen Ausgaben von Blatt 314 der Siegfriedkarte im Suchresultat zu erhalten, muss nach Kartenblättern mit dem Titel „Murten“ oder „Morat“ gesucht werden. Da jedoch im Titel von Blatt 312 der Begriff „Lac de Morat" enthalten ist, muss das Blatt Sugiez von der Suche ausgeschlossen werden. Dies ergibt folgende Zeichenkette:

http://auriga.admin.ch:8081/cgi-bin/vtls.web.gateway52?searchexp=\%28w\%3D bv80033582 $\% 29+$ AND $+\% 28 \% 28 \mathrm{t} \% 3$ Dmurten $\% 29+$ OR $+\% 28 \mathrm{t} \% 3$ Dmorat $\% 29 \% 2$ $9+$ NOT $+\% 28 \mathrm{t} \% 3$ Dsugiez $\% 29 \& f$ _lang $=\& f$ format $1=5 \& f \_$pub_start $=\& f$ pub_end $=$ $\&$ f_group $=0000 \&$ lang $=$ german

Das Ziel, mit verweis-sensitiven Blattübersichten einen leicht verständlichen und zielgerichten Zugang zu den Kartenbeständen zu schaffen wurde damit sicher erreicht. Der Zugriff erfolgt per Mausklick und es sind keine besonderen Vorkenntnisse im Hinblick auf Suchstrategien in einem Bibliothekskatalog erforderlich. Das neu geschaffene Angebot wird seither auch, wie erwartet, aktiv genutzt. Im Hinblick auf die Nutzung der Kartensammlung ist dabei folgendes beizufügen (s.a. die entsprechenden Webseiten $<$ http://www.swisstopo.ch/de/maps/ak/INDEX.htm>): 
Die alten Karten können nicht ausgeliehen werden. Ein Besuch vor Ort ist - nach Voranmeldung - für wissenschaftliche Zwecke möglich. Das Bundesamt für Landestopographie kann zwar keine alten Karten mehr verkaufen. Auf Wunsch werden jedoch Kopien oder hochqualitative Reproduktionen in analoger bzw. digitaler Form hergestellt, wobei Bestellungen schriftlich aufzugeben sind. Die Verrechnung erfolgt nach Tarif (zum Teil nach Aufwand).

\section{Ausblick}

Für das Projekt „Katalog Kartenarchiv“ stellt die Bereitstellung von verweis-sensitiven Blattübersichten der schweizerischen Landeskartenwerke einen bedeutenden Zwischenschritt dar. Mit den Landeskartenwerken wurde jedoch erst ein Teil der Kartensammlung in der Datenbank erschlossen, die weiter ausgebaut wird. Durch eine Schenkung gelangte zudem die bedeutende und umfangreiche Kartensammlung von Alfred Oberli in das Bundesamt für Landestopographie, die nun ebenfalls in die Erschließung einbezogen werden konnte. Schließlich wird auch der Aufbau einer Bilddatenbank angestrebt, wozu jedoch noch die erforderlichen Entscheide ausstehen. 


\section{WWW-Adressen (URLs)}

Digitale Karten - eine Herausforderung für die Kartensammlung? (H. Asche) http://www.alexandria.ucsb.edu/ - Alexandria Project http://libraries.mit.edu/research/ask/ask-general.html - Ask us live http://www.ifla.org - IFLA http://atlas.gc.ca - The national atlas of Canada online

Digitale Angebote in Kartenbibliotheken - vom Konzept zur Realisierung (J. Bühler) http://www.maps.ethz.ch - The World of Maps - Die Welt der Karten http://www.ethbib.ethz.ch/ks/ - Kartensammlung ETH Zürich http://www.maps.ethz.ch/imhof.html - Virtual Library Eduard Imhof

CD-ROM-Produkte in der Kartensammlung der ETH Zürich (H. Meyer) http://www.ethbib.ethz.ch/ks/cd-rom.html - CD-ROM-Bestand der ETH-Kartensammlung

Die Nutzung von GIS-Produkten in der Kartensammlung (J. Bühler) http://www.kogis.ch/sikgis_d.htm - SIK-GIS-Dateninventar

Verfilmung und Digitalisierung der Sammlung Ryhiner (T. Klöti) http://www.stub.unibe.ch/stub/ryhiner/ry-dt1.html - Ryhiner-Kartensammlung

Das Projekt "Elektronisches Kartenarchiv" an der UB Sachsen-Anhalt in Halle (J.-P. Grell) http://haweb1.bibliothek.uni-halle.de/cgi-bin/wwwlibmenu - OPAC Halle http://www.bibliothek.uni-halle.de/karten/ - Kartensammlung Halle http://www.gbv.de/ - GBV Gemeinsamer Bibliotheksverbund http://karten.bibliothek.uni-halle.de/mapwork/Sachsen-Anhalt/Eingangsbild/ - Sachsen-Anh . http://haweb1.bibliothek.uni-halle.de/cgi-bin/nphwwp3?DB=BES1.SYS8\&LANG=

DU\&EXT $=\mathrm{ON} \& \mathrm{CMD}=\mathrm{f}+\mathrm{swo}+\mathrm{emap}-$ Digitaler Kartenbestand

Der digitale, historische Kartenkatalog der SuUB Bremen (T. Piezunka + A. Kintrup) http://gauss.suub.uni-bremen.de - Historischer Kartenkatalog der SuUB Bremen http://www.internetvisor.de - Internet visor von megatel

Die "Virtual Library Eduard Imhof" - Kartographiegeschichte im Internet (K. Turconi) http://www.maps.ethz.ch/imhof.html - Virtual Library Eduard Imhof, deutsch http://www.maps.ethz.ch /imhof_engl.html - Virtual Library Eduard Imhof, englisch

Verweis-sensitive Indexblätter als Bestandesnachweis im Internet (W. Crom) http://www.dbiberlin.de/dbi_pub/bd_art/bd 99/99_04_07.htm

Artikel in Bibliotheksdienst 33, 1999 http://www.ethbib.ethz.ch/ks/index-bw50.html - Verweis-sensitives Indexblatt http://www.ethbib.ethz.ch/ks/tk50bw.html - Bestandesliste Baden-Württemberg 1:50.000 
Vom OPAC zum Indexblatt, vom SWB zur Online-Systematik (H. Wiesenmüller) http://www.wlb-stuttgart.de/referate/kartograph/bestand.htm - Internet-Kartenkatalog Crom http://db1-www.sub.uni-goettingen.de/gok/ - Göttinger Online-Klassifikation

\section{Von der verweis-sensitiven Blattübersicht zur Titelaufnahme im Bibliotheks.-Verbundkatalog (T. Klöti) \\ http://www.ethbib.ethz.ch/ks/indexblaetter.html - Beispiel Indexkarten http://www.swisstopo.ch/de/maps/ak/INDEX.htm - Kartensamml. Schweiz. Landestopograph. http://auriga.admin.ch:8081/ - Verbundkatalog Alexandria}

\section{Kartensammlung und Internet (J. Bühler)}

http://www.ethbib.ethz.ch/ks/ - Kartensammlung ETH Zürich http://www.ethbib.ethz.ch - ETH-Bibliothek Zürich http://oddens.geog.uu.nl/index.html - Oddens: The Fascinating World of Maps http://www.maps.ethz.ch - The World of Maps - Die Welt der Karten http://opac.nebis.ch:4505/ALEPH/ - Bibliotheksverbundkatalog NEBIS http://www.ethbib.ethz.ch/ks/indexblaetter.html - Indexblätter und Bestandeslisten http://www.ethbib.ethz.ch/ks/cd-rom.html - CD-ROM-Bestand http://www.maps.ethz.ch/gdc-education.html - Bildung für Kartenkuratoren im Internet http://www.maps.ethz.ch/imhof.html - Virtual Library Eduard Imhof

\section{"Die Welt der Karten" - ein Portal zur Suche nach Karten im Internet (J. Bühler)} http://www.maps.ethz.ch - The World of Maps - Die Welt der Karten http://oddens.geog.uu.nl/index.html - Oddens: The Fascinating World of Maps http://www.lib.utexas.edu/maps/index.html - Perry-Castañeda Library Map Collection http://alexandria.sdc.ucsb.edu/other-sites/ - Alexandria Digital Library http://www.cgrer.uiowa.edu/servers/servers_references.html - Maps+References, Univ.Iowa

\section{Metadatenkatalog für Raumdaten - Beispiel Geo-Guide (M. Schüler) http://www.sub.uni-goettingen.de - Niedersächs.Staats- u. Universitätsbibliothek http://www.Geo-Guide.de/ - Geo-Guide http://www.MathGuide.de/ - MathGuide http://www.sub.uni-goettingen.de/vlib/ -AngloAmericanGuide http://www.virtuellefachbibliothek.de - Virtuelle Forschungsbibliothek der DFG http://www.renardus.org/ - Renardus- The clever route to information http://www.oclc.org/connexion/ - OCLC CORK: Boost your library's net value http://scout.cs.wisc.edu/research/SPT/main.html - Scout Research}

\section{Wissen für Kartenkuratoren - aus dem Internet (J. Bühler)} http://www.maps.ethz.ch/gdc-education.html - LIBER Groupe des Cartothécaires - Eduction http://www.maps.ethz.ch/gdc-education2.html - Literatur zum Kartenbereich http://www.maps.ethz.ch/gdc-education4.html - GdC-Campus - Tutorials and Courses http://www.maps.ethz.ch/gdc-education6.html - Who is Who in Map Librarianship http://www.kb.nl/infolev/liber/intro.htm - LIBER Groupe des Cartothécaires 


\section{Autorenverzeichnis}

\section{Markus APPENZELLER}

Kartensammlung, ETH-Bibliothek, Rämistrasse 101, CH-8092 Zürich

Tel: +41 (1) $6322190 \quad$ E-mail: appenzeller@library.ethz.ch

Hartmut ASCHE, Prof. Dr.

Lehrstuhl Geoinformatik am Institut für Geographie, Univ. Potsdam, D-14469 Potsdam

Tel: +49 (31) $9772274 \quad$ E-mail: gislab@rz.uni-potsdam.de

Kurt BRUNNER, Prof. Dr.

Lehrstuhl für Kartographie und Topographie, Universität der Bundeswehr München,

D-85577 Neubiberg

Tel.: +49 (89) 6004-4049Ｅ-mail: kurt.brunner@unibw-muenchen.de

Jürg BÜHLER, Dr.

Kartensammlung, ETH-Bibliothek, Rämistrasse 101, CH-8092 Zürich

Tel: +41 (1) $6322184 \quad$ E-mail: juerg.buehler@library.ethz.ch

\section{Wolfgang CROM}

Kartenabteilung, Staatsbibliothek zu Berlin, Unter den Linden 8, D-10117 Berlin

Tel: +49 (30) $2661235 \quad$ E-mail: wolfgang.crom@sbb.spk-berlin.de

Jens-Peter GRELL

Kartenabteilung, Staatsbibliothek zu Berlin, Potsdamer Str. 33, D-10785 Berlin

Tel: +49 (345) $5522129 \quad$ E-mail: jens-peter.grell@sbb.spk-berlin.de

Andrea KINTRUP

Staats- und Universitätsbibliothek Bremen. Bibliothekstraße, 28359 Bremen

Tel: +49 (421) 218-2723Ｅ-mail: kintrup@suub.uni-bremen.de

Thomas KLÖTI, Dr.

Bundesamt für Landestopographie, Seftigenstrasse 264, CH-3084 Wabern/Bern

Tel: +41 (31) $9632241 \quad$ E-mail: thomas.kloeti@lt.admin.ch

\section{Agnes KOVACS}

Inst. f. Publizistik, Wissenschaft und Medienforschung, Univ. Zürich, CH-8035 Zürich

Tel. +41 (1) 6344663

E-mail: agnes.kovacs@ipmz.unizh.ch

Joachim LAMATSCH

PF150, D-79001 Freiburg

Tel: +49 (761) 554409

E-mail: lamatsch.joachim@t-online.de 